\title{
The Value of Synthesizing Evidence to Inform Cancer Nursing
}

\author{
Noyes, Jane
}

\section{Cancer Nursing}

DOI:

10.1097/NCC.0000000000000824

Published: 31/05/2020

Peer reviewed version

Cyswllt i'r cyhoeddiad / Link to publication

Dyfyniad o'r fersiwn a gyhoeddwyd / Citation for published version (APA):

Noyes, J. (2020). The Value of Synthesizing Evidence to Inform Cancer Nursing. Cancer Nursing, 43(3), 173-176. https://doi.org/10.1097/NCC.0000000000000824

\footnotetext{
Hawliau Cyffredinol / General rights

Copyright and moral rights for the publications made accessible in the public portal are retained by the authors and/or other copyright owners and it is a condition of accessing publications that users recognise and abide by the legal requirements associated with these rights.

- Users may download and print one copy of any publication from the public portal for the purpose of private study or research.

- You may not further distribute the material or use it for any profit-making activity or commercial gain

- You may freely distribute the URL identifying the publication in the public portal ?
}

Take down policy

If you believe that this document breaches copyright please contact us providing details, and we will remove access to the work immediately and investigate your claim. 
Jane Noyes

\section{The value of synthesising evidence to inform cancer nursing}

Making best use of evidence to inform cancer nursing practice is a global priority. Synthesising evidence is an efficient way of maximising use of existing evidence and preventing research wastage by commissioning unwarranted new research. The importance of the systematic review to informing clinical decisions is signified by the establishment of global clinical guideline developers such as the World Health Organisation (https://www.who.int/publications/guidelines/en/), United States Agency for Healthcare Research and Quality (https://www.ahrq.gov/), National Health and Medical Research Council of Australia (https://www.nhmrc.gov.au/), and the National Institute for Health and Care Excellence in the UK (https://www.nice.org.uk/). The entire clinical guideline development process is predicated on the systematic review of evidence from which recommendations for practice can be made. The field of cancer has also benefitted from national and international consensus statements on treatments and interventions drawing on systematic reviews in combination with clinical expertise and patient preferences.

Cancer was one of the first clinical specialities to embrace quantitative systematic reviews of the effects of drugs and other types of treatments. The Cochrane library for example, has more intervention effect reviews on cancer than any other topic. The large number of systematic reviews on cancer topics have subsequently been used to underpin clinical guideline development to transform the treatment options, associated nursing care, and improved outcomes for patients. Of specific interest, recent developments include a review to establish the effectiveness and value of European cancer nursing, which is one of the first of its type. ${ }^{1}$

Whilst the Cochrane-type of quantitative intervention effect review has achieved a state of supremacy, over the last 20 years there has been prolific development of other review methodologies to address different types of questions with diverse types of evidence (such as qualitative and mixed-method). Interestingly, nurses have been highly influential in the methodological development of diverse review types that are more likely to be useful in developing new theory and new insights into patient experience and nursing care. The new Cochrane Handbook, for example, includes a chapter on qualitative evidence synthesis ${ }^{2}$, and Cochrane has an Effective Practice and Organisation of Care review group (https://epoc.cochrane.org/about-us). In a more general context (see Figure 1), it is now possible to use diverse evidence synthesis methods for a much wider set of purposes, such as to:

- Determine the pool of known evidence on a topic

- Formulate review questions/determine outcomes and clarify review parameters

- Clarify concepts and synthesise theory

- Synthesise policy intentions and outcomes

- Synthesise system wide policy outcomes

- Develop theory to inform a primary study

- Develop theory as a primary purpose

- Understand illness experiences

- Determine how promising practices work

- Understand patient, carer and key stakeholder experiences, values and preferences concerning interventions

- Determine factors that impact on intervention implementation, fidelity, reach, acceptability, feasibility, and to identify benefits and harms

- Estimate the cost and effectiveness of interventions 
- Determine prognosis

- Determine diagnostic test accuracy

- Determine the psychometric properties of instruments

- Determine the effects and impacts of complex, health system wide interventions

- Integrate quantitative and qualitative evidence

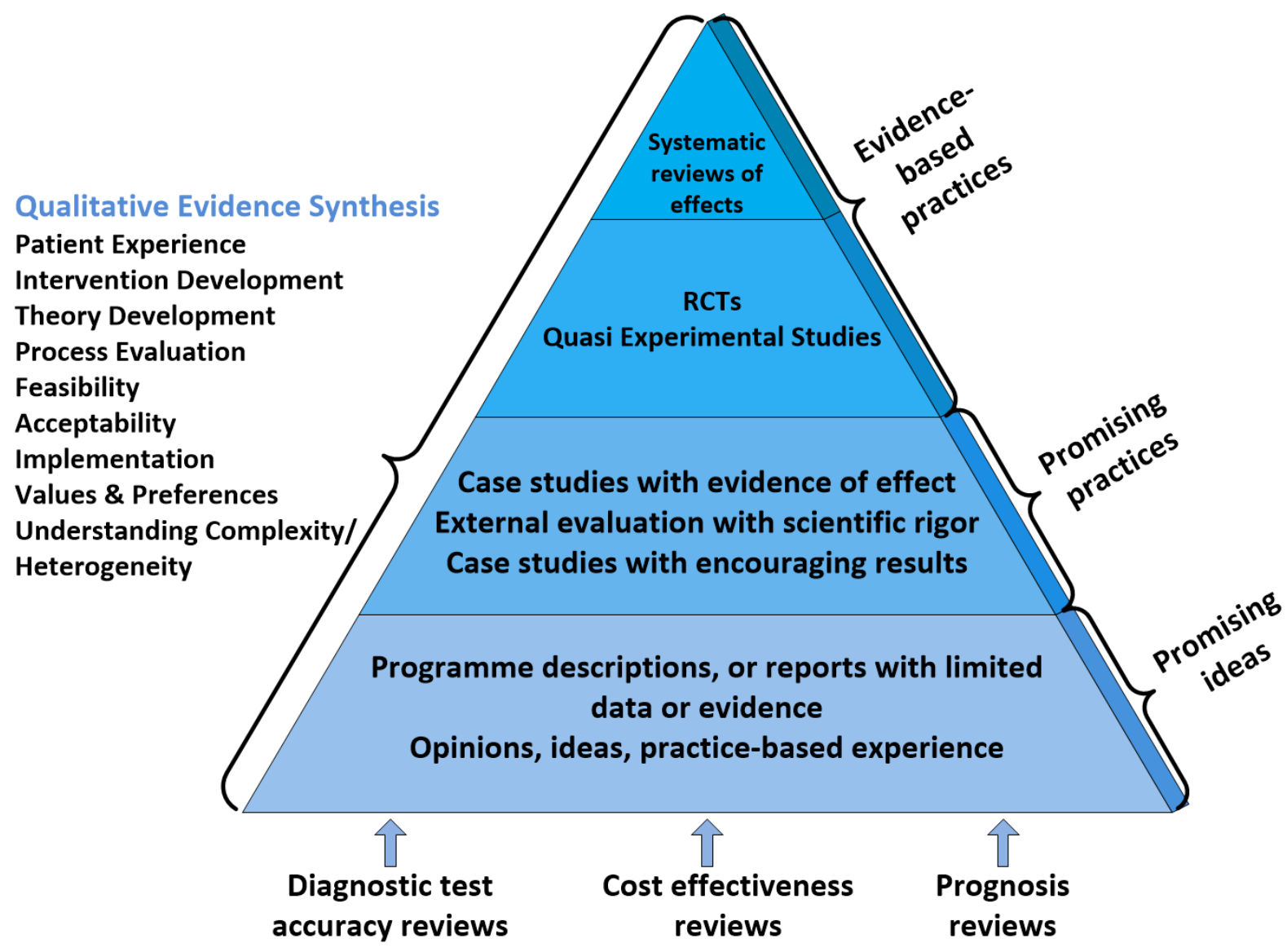

Figure 1. New hierarchy of evidence that responds to user requirements for the inclusion of diverse evidence for decision-making and timely reviews. Adapted from

https://www.homelesshub.ca/sites/default/files/attachments/PPFramework Part1.pdf

Guideline developers and decision makers increasingly require qualitative and mixed method syntheses as well as reviews of intervention effects, diagnostic test accuracy and prognosis to populate specific aspects of the 'evidence to decision' framework ${ }^{3}$, such as patient values, preferences and experiences, feasibility, implementation and resource considerations, and equity implications (see Figures 1 and 2). It is these specific phenomena that can be addressed by newer review types and methods to better inform cancer nursing and underpin guideline development. 


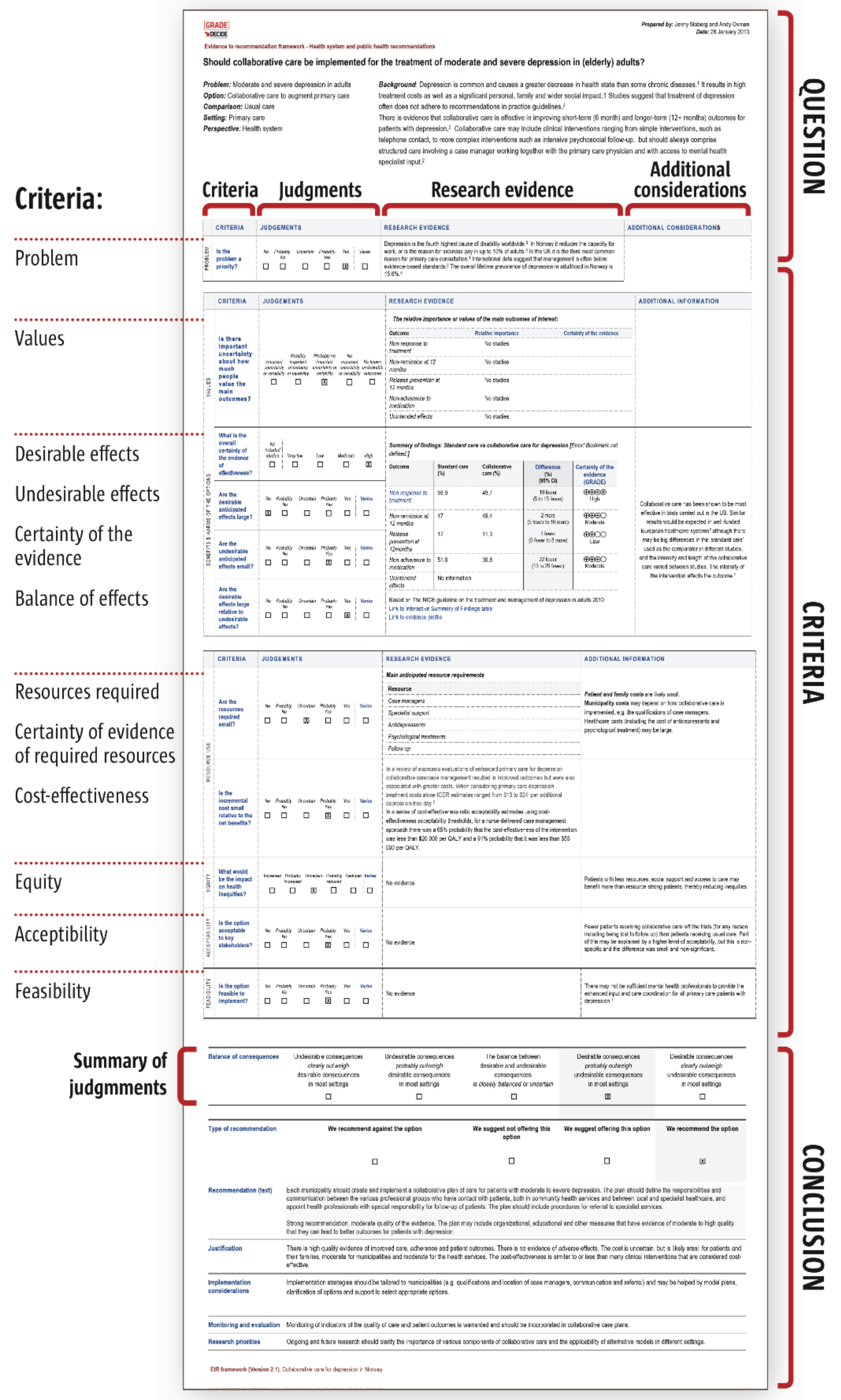

Figure 2. The DECIDE Framework. Courtesy of Simon Lewin. 
The majority of published contemporary reviews in cancer nursing however use very few of the many available newer methods, especially those qualitative evidence synthesis methods that are designed to advance new theory and theoretical insights that go beyond the primary studies. ${ }^{4}$ There are currently, for example, around 18 different and sometimes overlapping qualitative evidence synthesis methods that vary in complexity. ${ }^{5-7}$ Reviewers commonly find it difficult to select an appropriate synthesis methodology for their specific context. Reviewers also tend to stick to the same methodology that they are familiar with, rather than consider the best methodology for the type of available evidence. To support reviewers to make the best choice, Booth and colleagues have produced the RETREAT checklist of things to consider when selecting a methodology. ${ }^{5-6}$

Box 1. The RETREAT Framework for selecting an appropriate methodology. Reproduced from Booth et al. ${ }^{5-}$ 6

\begin{tabular}{|l|l|}
\hline $\mathbf{R}$ & Review question \\
\hline $\mathbf{E}$ & Epistemology \\
\hline $\mathbf{T}$ & Time/time frame \\
\hline $\mathbf{R}$ & Resources \\
\hline $\mathbf{E}$ & Expertise \\
\hline $\mathbf{A}$ & Audience and purpose \\
\hline $\mathbf{T}$ & Type of data \\
\hline
\end{tabular}

Many cancer interventions that involve nursing also tend to be 'complex interventions' and there is less (although growing) experience of undertaking mixed-method reviews of complex interventions that focus on complexity and involve health systems level change. ${ }^{4-8}$ The use of theory to design reviews and interpret evidence is also increasingly used to help review authors to produce a more theory informed and useful product for decision-making. Theory in the form of logic models and social theories can help structure and focus a systematic review of any design and can be used as an integrative or interpretive lens. Cochrane has produced detailed guidance on the choice of theory for use in systematic reviews. ${ }^{9}$

Those reviewers that do apply newer and more novel methods frequently find it challenging to interpret and apply the evidence synthesis methods and tools as intended by the originators. ${ }^{10-11}$ Whilst acknowledging that funded reviews often need to be undertaken rapidly, there appears to be a lot of confusion about synthesis methods and designs, blurring of different methods, short cuts being taken and missing out of important stages and processes when it is not appropriate to do so. Reviewers have also found it challenging to report their review in a way that has maximum utility for decision-makers. ${ }^{10}$ In recent years methodologists have recognised that too many qualitative evidence syntheses were poorly reported and thus could not be used to make decisions, and responded by developing detailed reporting guidelines to support both the better conduct and reporting of generic qualitative evidence syntheses and meta-ethnographies. ${ }^{12-13}$ Likewise many reports of meta-analyses do not meet the PRISMA reporting requirements, and reports of quantitative syntheses without meta-analysis have been particularly poor, 
leading to the new (SWiM) reporting guidance which is an extension of PRISMA. ${ }^{14-15}$ There is at present no specific reporting guideline for mixed-method reviews, but Flemming and colleagues outline some principles to follow. ${ }^{16}$ Other recent developments include GRADE CERQual to assess the confidence in synthesised qualitative findings. ${ }^{17}$ This latter development is important as decision makers have got used to the similar GRADE method (https://www.gradeworkinggroup.org/) for assessing the certainty of evidence of intervention effects and were keen to have a similar system for qualitative evidence syntheses. Syntheses of qualitative evidence are of more value to decision makers if they have confidence in the quality of the review and the strength of the evidence.

Of particular concern, with some notable exceptions, patient and public involvement has been much slower to be fully integrated into the conduct of evidence syntheses, especially those reviews that are not funded. Many reviews are conducted without any patient and public involvement, whereas for most funded reviews their input is expected because the review product is likely to be more patient-centred and of greater value to decision makers if they are co-produced. Cochrane for example has a huge consumer network of people to drawn on. Many local not for profit organisations and individuals are however more than willing to contribute to non funded reviews because they want to see improved treatments and services for people with cancer. There is also a big evidence gap in the conduct of reviews at the interface between health and social cancer care, and reviews focused purely on social care and cancer. People living with cancer experience a myriad of psychological, social and domestic problems that impact on their life and wellbeing. Cancer nurses are well placed to fill this known evidence gap to benefit patients.

It is however positive to see a mixture of different review types and designs in the current themed issue on evidence synthesis. Selected reviews include a scoping review, priority setting review, meta-analyses and qualitative evidence syntheses addressing various questions of importance to cancer nursing. Of particular interest, Bernier Carney et $\mathrm{al}^{18}$ use meta-ethnography to transform the findings of primary qualitative studies to better understand the experiences during childhood cancer survivorship. Meta-ethnography is one of the more complex qualitative evidence synthesis methods and requires experience of conducting primary qualitative research to fully utilise the power of the methodology to develop new theory and interpretations that move beyond the primary study findings. Cadorin et al ${ }^{19}$ undertook a mixed-method review and published their protocol in PROSPERO (International prospective register of systematic reviews https://www.crd.york.ac.uk/PROSPERO/). It is a marker of best practice to make publicly available the review protocol prior to conducting the review. Although the summary of findings is articulated very briefly and not in a way that was originally intended, it is good to see that Diaw et al ${ }^{20}$ applied GRADE CERQual to assess the confidence in their synthesised qualitative findings. Presenting a summary of findings table with associated assessments of confidence can be exceptionally helpful for decision-makers. It was also encouraging to see Han et $\mathrm{al}^{21}$ use a symptom management theory as the theoretical framework to inform the design and interpretation of their quantitative review and meta-analysis.

In summing up the current state of the art of evidence synthesis in the first two decades of the $21^{\text {st }}$ century, the best one can say is that it is a mixed-picture of great progress and unfulfilled potential. There are different evidence synthesis methods for varying purposes that are continuing to evolve. Global evidence synthesis producers, guideline producers and decision makers are now much more aware of the value of syntheses of diverse evidence types. There is further potential for cancer nurses to embrace the full range of synthesis methods available in order to make best use of the available evidence in health and social care. But, they need to apply evidence synthesis methods carefully and rigorously to produce higher quality reviews that are valued and used by decision-makers. There is now much better methodological 
guidance to support the conduct and reporting of reviews to further improve their quality and utility for decision-making.

\section{References}

1. Campbell, Pauline, Torrens, Claire, Kelly, Daniel, Charalambous, Andreas, Domenech-Climent, Nuria, Nohavova, Iveta, Östlund, Ulrika, Patiraki, Elisabeth, Salisbury, David, Sharp, Lena, Wiseman, Theresa, Oldenmenger, Wendy and Wells, Mary 2017. Recognising European Cancer Nursing: Protocol for a systematic review and meta-analysis of the evidence of effectiveness and value of cancer nursing. Journal of Advanced Nursing 73 (12) , pp. 3144-3153. 10.1111/jan.13392

2. Noyes J, Booth A, Cargo M, Flemming K, Harden A, Harris J, Garside R, Hannes K, Pantoja T, Thomas J. Chapter 21: Qualitative evidence. In: Higgins JPT, Thomas J, Chandler J, Cumpston M, Li T, Page MJ, Welch VA (editors). Cochrane Handbook for Systematic Reviews of Interventions version 6.0 (updated July 2019). Cochrane, 2019. Available from www.training.cochrane.org/handbook.

3. DECIDE Evidence to Decision Framework, 2015. Available from: http://www.decidecollaboration.eu/evidence-decision-etd-framework

4. Flemming K, Booth A, Garside R, Tunçalp Ö, Noyes J. Qualitative evidence synthesis for complex interventions and guideline development: clarification of the purpose, designs and relevant methods. BMJ Glob Health. 2019 Jan 25;4(Suppl 1):e000882. doi: 10.1136/bmjgh-2018-000882. eCollection 2019. Review.

5. BOOTH, A., NOYES J, FLEMMING K, GERHARDUS, A., WAHLSTER, P., VAN DER WILT, G.J., MOZYGEMBA, K., REFOLO, P., SACCHINI, D., TUMMERS, M., REHFUESS, E. (2016) Guidance on choosing qualitative evidence synthesis methods for use in health technology assessments of complex interventions [Online]. Available from: http://www.integrate-hta.eu/downloads/

6. Booth A, Noyes J, Flemming K, Gerhardus A, Wahlster P, van der Wilt GJ, Mozygemba K, Refolo P, Sacchini $D$, Tummers $M$, Rehfuess E. Structured methodology review identified seven (RETREAT) criteria for selecting qualitative evidence synthesis approaches. J Clin Epidemiol. 2018 Jul;99:41-52. doi: 10.1016/j.jclinepi.2018.03.003. Epub 2018 Mar 13. Review

7. Booth A, Noyes J, Flemming K, Moore G, Tunçalp Ö, Shakibazadeh E. Formulating questions to explore complex interventions within qualitative evidence synthesis. BMJ Glob Health. 2019 Jan 25;4(Suppl 1):e001107. doi: 10.1136/bmjgh-2018-001107. eCollection 2019.

8. Noyes J, Booth A, Moore G, Flemming K, Tunçalp Ö, Shakibazadeh E. Synthesising quantitative and qualitative evidence to inform guidelines on complex interventions: clarifying the purposes, designs and outlining some methods. BMJ Glob Health. 2019 Jan 25;4(Suppl 1):e000893. doi: 10.1136/bmjgh-2018-000893. eCollection 2019.

9. Noyes J., Hendry M., Booth A., Chandler J., Lewin S., Glenton C. \& Garside R. (2016) Current use was established and Cochrane guidance on selection of social theories for systematic reviews of complex interventions was developed. Journal of Clinical Epidemiology pii, S0895-4356(16)00005-6. doi: 10.1016/j.jclinepi.2015.12.009.

10. France EF, Ring N, Thomas R, Noyes J, Maxwell M, Jepson R.A methodological systematic review of what's wrong with meta-ethnography reporting. BMC Med Res Methodol. 2014 Nov 19;14:119. doi: 10.1186/1471-2288-14-119. Review.

11. France EF, Uny I, Ring N, Turley RL, Maxwell M, Duncan EAS, Jepson RG, Roberts RJ, Noyes J. A methodological systematic review of meta-ethnography conduct to articulate the complex analytical phases. BMC Med Res Methodol. 2019 Feb 18;19(1):35. doi: 10.1186/s12874-019-0670-7.

12. Tong, A., Flemming, K., Mclnnes, E. et al. Enhancing transparency in reporting the synthesis of qualitative research: ENTREQ. BMC Med Res Methodol 12, 181 (2012).

https://doi.org/10.1186/1471-2288-12-181

13. France EF, Cunningham M, Ring N, Uny I, Duncan EA, Jepson RG, Maxwell M, Roberts RJ, Turley RL, Booth A, Britten N, Flemming K, Gallagher I, Garside R, Hannes K, Lewin S, Noblit GW, Pope C, Thomas J, Vanstone M, Higginbottom GMA, Noyes J. Improving reporting of meta-ethnography: 
The eMERGe reporting guidance. J Adv Nurs. 2019 May;75(5):1126-1139. doi: 10.1111/jan.13809. Epub 2019 Jan 15

14. Liberati Alessandro, Altman Douglas G, Tetzlaff Jennifer, Mulrow Cynthia, Gøtzsche Peter $C$, loannidis John P A et al. The PRISMA statement for reporting systematic reviews and metaanalyses of studies that evaluate healthcare interventions: explanation and elaboration BMJ 2009; 339 :b2700

15. Campbell Mhairi, McKenzie Joanne E, Sowden Amanda, Katikireddi Srinivasa Vittal, Brennan Sue E, Ellis Simon et al. Synthesis without meta-analysis (SWiM) in systematic reviews: reporting guideline BMJ 2020; $368: 16890$

16. Flemming K, Booth A, Hannes K, Cargo M, Noyes J. Cochrane Qualitative and Implementation Methods Group guidance series-paper 6: reporting guidelines for qualitative, implementation, and process evaluation evidence syntheses. J Clin Epidemiol. 2018 May;97:79-85. doi: 10.1016/j.jclinepi.2017.10.022. Epub 2017 Dec 6

17. Lewin S, Booth A, Glenton C, Munthe-Kaas H, Rashidian A, Wainwright M, Bohren MA, Tunçalp Ö, Colvin CJ, Garside R, Carlsen B, Langlois EV, Noyes J. Applying GRADE-CERQual to qualitative evidence synthesis findings: introduction to the series. Implement Sci. 2018 Jan 25;13(Suppl 1):2. doi: 10.1186/s13012-017-0688-3.

18. Bernier Carney K, Guite J, Young E, Starkweather E. Forced Enlightenment A Metasynthesis of Experiences During Childhood Cancer Survivorship. Cancer Nursing Vol.00,No.0,2019

19. Cadorin L, Bressan, V, Truccolo I, Suter N. Priorities for Cancer Research From the Viewpoints of Cancer Nurses and Cancer Patients A Mixed-Method Systematic Review. Cancer Nursing Vol.00,No.0,2019.

20. Diaw M, Sibeoni J Manolios E, Gouacide J-M,, Brami C, Verneuil, L, Revah-Levy, A. The Lived Experience of Work-Related Issues Among Oncology Nurses A Metasynthesis. Cancer Nursing Vol.00,No.0,2019

21. Han CJ, Yang GS, Syrjala K. Symptom Experiences in Colorectal Cancer Survivors After Cancer Treatments A Systematic Review and Meta-analysis Cancer Nursing Vol.00,No.0,2019 